\title{
Chromatin occupancy of transcription factor 7-like 2 (TCF7L2) and its role in hepatic glucose metabolism
}

\author{
L. Norton • M. Fourcaudot • M. A. Abdul-Ghani • \\ D. Winnier • F. F. Mehta $\cdot$ C. P. Jenkinson • \\ R. A. DeFronzo
}

Received: 29 April 2011 / Accepted: 18 July 2011 /Published online: 7 September 2011

(C) Springer-Verlag 2011

\begin{abstract}
Aims/hypothesis The mechanisms by which transcription factor 7-like 2 (TCF7L2) regulates the pathways that are important in the pathogenesis of type 2 diabetes are unknown. We therefore examined the role of TCF7L2 in hepatic glucose production (HGP) in vitro and characterised the wholegenome chromatin occupancy of TCF7L2 in hepatocytes. Methods We investigated the effect of TCF7L2 silencing and overexpression on HGP from gluconeogenic precursors and used chromatin-immunoprecipitation (ChIP) combined with massively parallel DNA sequencing (ChIP-Seq) to investigate the DNA binding patterns of TCF7L2 across the whole genome. Results Silencing of TCF7L2 induced a marked increase in basal HGP, which was accompanied by significant increases in the expression of the gluconeogenic genes Fbpl, Pckl and G6pc. Overexpression of $T c f 7 l 2$ reversed this phenotype and significantly reduced HGP. TCF7L2 silencing did not affect the half-maximal inhibitory concentration of insulin or metformin, but HGP remained elevated in TCF7L2-silenced cells due to the increased baseline HGP. Using ChIP-Seq, we detected 2,119 binding events across the genome. Pathway analysis demonstrated that diabetes genes were significantly over-represented in the dataset. Our results indicate that TCF7L2 binds directly to multiple genes that are important in regulation of glucose metabolism in the liver, including Pck1, Fbp1, Irs1, Irs2, Akt2, Adipor1, Pdk4 and Cptla.
\end{abstract}

Electronic supplementary material The online version of this article (doi:10.1007/s00125-011-2289-z) contains peer-reviewed but unedited supplementary material, which is available to authorised users.

L. Norton $(\bowtie) \cdot$ M. Fourcaudot $\cdot$ M. A. Abdul-Ghani •

D. Winnier · F. F. Mehta • C. P. Jenkinson • R. A. DeFronzo

Diabetes Division, University of Texas Health Science Center,

7703 Floyd Curl Drive,

San Antonio, TX 78229, USA

e-mail: nortonl@uthscsa.edu
Conclusions/interpretation TCF7L2 is an important regulator of HGP in vitro and binds directly to genes that are important in pathways of glucose metabolism in the liver. These data highlight the possibility that TCF7L2 may affect fasting and postprandial hyperglycaemia in carriers of at-risk TCF7L2 genetic polymorphisms.

Keywords Hepatic glucose metabolism - TCF7L2 - Type 2 diabetes

$\begin{array}{ll}\text { Abbreviations } \\ \text { ChIP } & \text { Chromatin-immunoprecipitation } \\ \text { ChIP-Seq } & \text { ChIP combined with massively parallel } \\ & \text { DNA sequencing } \\ \text { FDR } & \text { False discovery rate } \\ \text { GAPDH } & \text { Glyceraldehyde-3-phosphate dehydrogenase } \\ \text { HGP } & \text { Hepatic glucose production } \\ \text { HNF4 } \alpha & \text { Hepatocyte nuclear factor 4 } \alpha \\ \text { IC } & \text { Half-maximal inhibitory concentration } \\ \text { IPA } & \text { Ingenuity Pathway Analysis } \\ \text { pAKT } & \text { Phosphorylated AKT } \\ \text { PDC } & \text { Pyruvate dehydrogenase complex } \\ \text { PDK4 } & \text { Pyruvate dehydrogenase kinase 4 } \\ \text { siRNA } & \text { Small interfering RNA } \\ \text { TCF7L2 } & \text { Transcription factor 7-like 2 } \\ \text { TSS } & \text { Transcriptional start site } \\ \text { WNT } & \text { Morphogenic wingless-type MMTV } \\ & \text { integration site family }\end{array}$

\section{Introduction}

The most significant type 2 diabetes candidate gene to emerge from recent genome-wide association studies is TCF7L2. Linkage analysis originally identified a type 2 
diabetes susceptibility locus on chromosome 10 [1], which was later refined to a single nucleotide polymorphism within intron 3 of TCF7L2 [2]. This association has since been replicated many times in several populations throughout the world [3]. The gene product, transcription factor 7like 2 (TCF7L2), is a high-mobility box-containing transcription factor that plays an important role in executing downstream signals of the morphogenic wingless-type MMTV integration site family (WNT) pathway. The WNT signalling cascade is a highly conserved developmental pathway, which, classically, has been associated with embryogenesis and tumorigenesis. Activation of the pathway leads to accumulation of $\beta$-catenin in the nucleus, which associates with the LEF/TCF family of transcription factors to regulate the transcription of WNT target genes, as detailed elsewhere [4]. Following the genetic association, numerous laboratories have attempted to describe a link between WNT signalling and the pathways involved in the aetiology of type 2 diabetes. Most of these studies have focused on the pancreatic beta cell, as early indications were that TCF7L2 is important in controlling glucose- and incretin-stimulated insulin secretion in vivo and in vitro [5].

However, several studies have indicated that TCF7L2 polymorphisms are also associated with elevated rates of hepatic glucose production (HGP) and reduced hepatic insulin sensitivity in humans in vivo $[6,7]$. In carriers of the at-risk T allele at rs7903146, rates of basal HGP were elevated and failed to be suppressed normally during a euglycaemic-hyperinsulinaemic $\left(40 \mathrm{mU} \mathrm{m}^{-2} \mathrm{~min}^{-1}\right)$ clamp [8].

The potential link between TCF7L2 and hepatic glucose metabolism is further strengthened by increasing evidence of a key role for the $\mathrm{WNT} / \beta$-catenin pathway in driving metabolic zonation in the liver [9]. Hepatocytes are metabolically specialised, depending partly on their position along the porto-central axis of the liver-cell plate [10]. Periportal hepatocytes in close proximity to the biliary duct, portal vein and hepatic artery are known to be gluconeogenic in nature and express key enzymes involved in this function. Hepatocytes with a more central location, designated as pericentral, are naturally glycolytic and display a gene expression profile more appropriate for this phenotype [11]. Functional zonation, therefore, is directed by the transcriptional regulation of genes that are specific to the function concerned. For example, key genes of gluconeogenesis $F B P 1, P C K 1$ and $G 6 P C$ are all upregulated in periportal hepatocytes. In contrast, glycolytic genes such as $G C K$ and $L D H D$ are overexpressed in pericentral hepatocytes [12]. Remarkably, inactivation of $\mathrm{WNT} / \beta$-catenin signalling in mouse liver is sufficient to transform hepatocytes from a pericentral to a periportal phenotype [13]. This metabolic switch is associated with dramatic changes in the expression of genes involved in gluconeogenesis, including Pckl and G6pc [14].

In the current study, we demonstrate that silencing of $T c f 7 l 2$ specifically in hepatocytes leads to a marked increase in HGP. We also provided evidence that this effect occurs primarily through increased expression of genes involved in gluconeogenesis. Using whole-genome chromatinimmunoprecipitation (ChIP) combined with massively parallel DNA sequencing (ChIP-Seq), we also document that TCF7L2 specifically binds to multiple genes that are important for the regulation of glucose metabolism in hepatocytes.

\section{Methods}

Cell culture The rat hepatoma cell line, H4IIE, was used in the present study and has been described in detail elsewhere [15]. H4IIE cells are highly differentiated, display a greater signal/noise ratio for HGP than other hepatic cell lines (i.e. HepG2, HuH6) and have been used extensively for the study of HGP in vitro [15-17]. Low passage cells were obtained from the American Type Culture Collection (ATCC, Manassas, VA, USA) and routinely cultured in DMEM supplemented with $4.5 \mathrm{mmol} / 1$ glucose and $10 \%$ (vol./vol.) FBS.

TCF7L2 silencing and glucose production assay We silenced TCF7L2 in H4IIE cells using the Neon Transfection System (Invitrogen, Carlsbad, CA, USA). Cells in suspension were electroporated (pulse voltage $1,300 \mathrm{~V}$, pulse width $30 \mathrm{~ms}$, pulse number 1) with $100 \mathrm{nmol}$ scrambled small interfering RNA (siRNA) sequence or $100 \mathrm{nmol}$ mouse Tcf7l2 siRNA (Dharmacon SMARTpool, Lafayette, CO, USA). To ensure that all potential TCF7L2 splice variants were targeted, we used a pool of siRNA sequences that were complementary to various regions of the open reading frame (ORF) and 3' untranslated region (UTR). Following electroporation, cells were immediately plated for the glucose production assay. Electroporated cells were allowed to grow for $24 \mathrm{~h}$ before the medium was replaced with serum-, Phenol Red- and glucose-free DMEM supplemented with $20 \mathrm{mmol} / \mathrm{l} \mathrm{D}$-lactate and $2 \mathrm{mmol} / \mathrm{l}$ sodium pyruvate. This medium was left on the cells for $10 \mathrm{~h}$ before being replaced with identical medium, with or without additives (insulin and metformin), and left overnight for the glucose production assay. The medium was then assayed for glucose using a kit (Amplex Red Glucose Assay; Invitrogen). Using this technique, glucose production was measured between 24 and $48 \mathrm{~h}$ after electroporation, which was the period of maximal TCF7L2 silencing (Fig. 1a). For overexpression studies, full-length human TCF7L2-myc was obtained from B. Volgelstein via Addgene (Cambridge, MA, USA) [18]. 


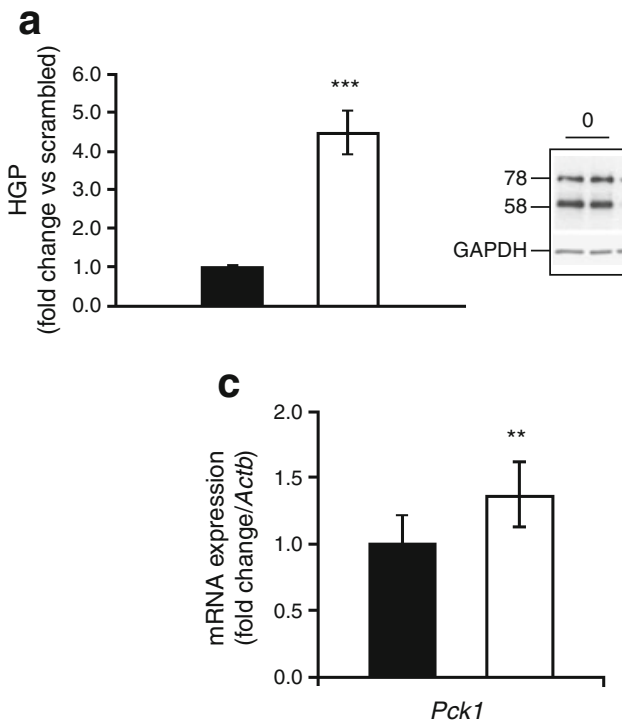

Fig. 1 a An siRNA time course experiment demonstrating the optimal silencing of TCF7L2 protein between 24 and $48 \mathrm{~h}$ after electroporation. During this period of maximal silencing, HGP from $20 \mathrm{mmol} / \mathrm{l}$ lactate and $2 \mathrm{mmol} / \mathrm{l}$ pyruvate was examined and found to be markedly increased. HGP determination was performed in triplicate wells for each treatment; results are representative of five independent experiments. Black bars, scrambled control; white bars, Tcf $7 l 2$ siRNA. b Overexpression of wild-type (WT) TCF7L2 reversed this phenotype

Western blotting After treatment, H4IIE cells were washed in ice-cold PBS and cellular protein was collected by scraping the cells into $50 \mu$ protein extraction buffer, after which western blotting was carried out as described [19]. Membranes were probed with the following antibodies, all supplied by Cell Signaling Technology (Danvers, MA, USA) unless stated otherwise: TCF7L2 (number 2569), total AKT (number 4691), phosphorylated AKT (pAKT) (number 4060), myc-tag (number 2278) and glyceraldehyde-3-phosphate dehydrogenase (GAPDH) (G9545; Sigma, St Louis, MO, USA).

Quantitative RT-PCR analysis Cells in 24-well plates were immediately washed with ice-cold PBS following treatment and lysed with $500 \mu \mathrm{l}$ Trizol reagent (Invitrogen). Total RNA was extracted, cDNA prepared and real-time PCR carried out as previously described [19]. TaqMan primer and probe pre-mixes were purchased (Applied Biosystems, Carlsbad, CA, USA). For ChIP assays, primers were designed for SYBR Green quantification, the sequences of which are listed in the electronic supplementary material (ESM Table 1).

ChIP-Seq ChIP DNA was prepared using a kit (number 9003; Enzymatic SimpleChIP Magnetic Kit; Cell Signaling) and anti-TCF7L2 antibody (number 2569; Cell Signaling). This antibody was designed against a peptide sequence surrounding Leu330 of the TCF7L2 protein. This

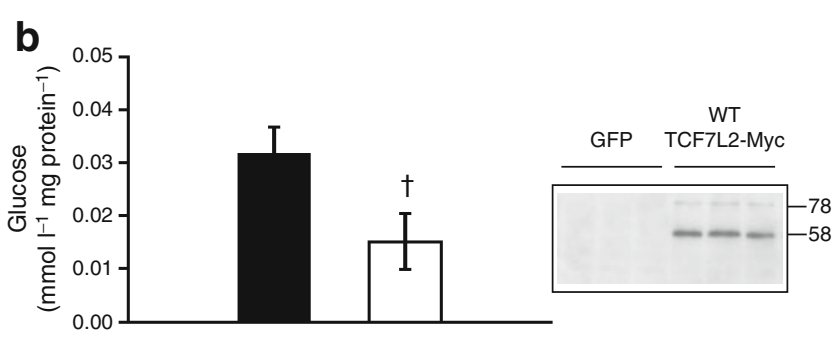

e

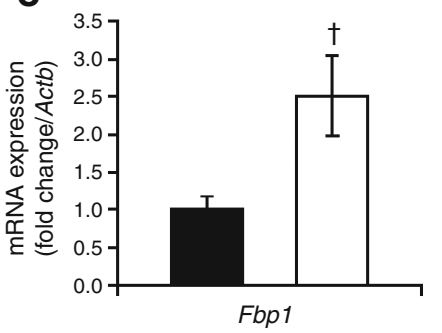

and led to a reduction in HGP. Black bars, green fluorescent protein (GFP) control plasmid; white bars, TCF7L2 WT plasmid. Silencing of TCF7L2 also increased the mRNA levels of several key genes involved in gluconeogenesis, including Pck1 (c), G6pc (d) and Fbpl (e). Gene expression analysis ( $n=12$ wells/treatment) was performed at the end of a silencing and HGP experiment, and is representative of duplicate independent gene expression experiments. Values are presented as mean $\pm \mathrm{SD} ; * * p<0.01, * * * p<0.001,{ }^{\dagger} p<0.0001$ vs control

residue is at the $3^{\prime}$ end of exon 9 and represents a region of conservation in all Tcf7l2 mRNA transcripts [20, 21]. Sequencing libraries were prepared from 30 ng ChIP DNA using a kit (ChIP-Seq Library Preparation; Illumina, San Diego, CA, USA). The TCF7L2 library was prepared from DNA obtained from replicate ChIP reactions $(n=4)$.

ChIP-Seq data analysis: identification of TCF7L2-enriched regions Analysis of ChIP-Seq data was performed with CLC Genomics Workbench version 4.5.1 (Cambridge, MA, USA). Raw 40 bp reads for the input control $(29,226,566$ reads $)$ and TCF7L2 ChIP reaction (30,688,073 reads) were first mapped to the repeat-masked Rat Reference (RN4) Genome (UCSC Genome Browser, http://genome.ucsc.edu/, accessed 28 September 2010). ChIP-Seq peak detection was then carried out using a window size of $250 \mathrm{bp}$ and a false discovery rate (FDR) of $1 \%$.

Motif analysis We searched for occurrence of the canonical (T/A-T/A-C-A-A-A-G) or evolutionarily conserved (A-C/G-T/A-T-C-A-A-A-G) TCF7L2 binding motif in each peak ( \pm 400 bp flanking DNA). To perform a bias-free search for novel and potentially unknown TCF7L2 motifs in the rat, we also used MEME-ChIP [22] on a small subset of the ChIP peaks. For this analysis, each peak was extracted with $25 \mathrm{bp}$ of the $5^{\prime}$ and $3^{\prime}$ flanking sequences, and the analysis was run with default conditions, but with a minimum and maximum motif width of 6 and 15 respectively. 
Statistical analysis Data are presented as means \pm SEM. Two-tailed $t$ tests were used to detect statistical differences in cell culture studies.

\section{Results}

TCF7L2 and glucose production We initially determined that TCF7L2 was highly abundant in the hepatic cell line H4IIE (Fig. 1a). We were able to silence TCF7L2 protein production by over $70 \%$ and $80 \%$ for the 78 and $58 \mathrm{kDa}$ bands, respectively (Fig. 1a). Protein levels began to recover $48 \mathrm{~h}$ after silencing, returning to near normal levels after $96 \mathrm{~h}$. Notably, we did not observe any additional protein isoforms that were indicative of alternative splicing. We also did not detect any effect of TCF7L2 silencing on cell proliferation as measured by the 3-(4,5-dimethylthiazol-2-yl)-5-(3-carboxymethoxyphenyl)-2-(4-sulfophenyl)-2H-tetrazolium (MTS) assay (ESM Fig. 1).

In rat hepatocytes in which TCF7L2 was silenced, addition of lactate/pyruvate caused a three- to fivefold increase in glucose production compared with the scrambled siRNA control (Fig. 1a). This effect was highly reproducible and was reversed when wild-type TCF7L2 was overproduced. Overabundance of myc-tagged TCF7L2 in hepatocytes induced a $50 \%$ reduction in glucose production determined over a $6 \mathrm{~h}$ period (Fig. 1b).

Expression of genes involved in gluconeogenesis The mRNA expression of a number of key gluconeogenic enzymes was also increased significantly in TCF7L2-silenced cells, when compared with the scrambled siRNA control (Fig. 1b). Pckl (36\%, $p<0.01$; Fig. 1c), Fbpl (152\%, $p<0.0001$; Fig. 1d) and G6pc (132\%, $p<0.0001$; Fig. 1e) were all significantly upregulated. The additional rate-controlling enzyme of gluconeogenesis, pyruvate carboxylase $(P c)$, was not affected by TCF7L2 silencing (ESM Fig. 2). Expression of Hnf4a was elevated following TCF7L2 silencing, whereas Ppargcla mRNA was unaffected (ESM Fig. 2).

Insulin and metformin sensitivity of glucose production We then investigated whether TCF7L2 silencing affected the sensitivity of HGP to insulin and metformin. Glucose production from lactate/pyruvate in the hepatocytes was remarkably sensitive to insulin, with a half-maximal inhibitory concentration $\left(\mathrm{IC}_{50}\right)$ of $100 \mathrm{pmol} / \mathrm{l}$, whereas higher, but still physiological concentrations of metformin were required $\left(\mathrm{IC}_{50} 200 \mu \mathrm{mol} / \mathrm{l}\right)$. TCF7L2 silencing did not affect the sensitivity of HGP to insulin or metformin (Fig. 2a, b), although for any given insulin or metformin concentration, HGP was higher in TCF7L2silenced hepatocytes. Confirming the comparable sensi- tivity of $T c f 7 l 2$ siRNA and scrambled siRNA treated cells to insulin, serine phosphorylation of AKT2 by insulin was indistinguishable (Fig. 2c, d). The inability of insulin and metformin to overcome the effect of TCF7L2 silencing and return the rate of HGP to levels observed with the scrambled siRNA control may have been due to impaired suppression of Pckl (Fig. 2e) and G6pc (Fig. 2f) expression in TCF7L2-silenced cells compared with scrambled control cells.

ChIP assay validation To gain further insight into the molecular events underlying the changes in glucose metabolism, we determined the precise transcriptional targets of TCF7L2 in hepatocytes in vitro. First, we confirmed that the antibody was specific to TCF7L2 at the Axin2 promoter and compared this to enrichment of TCF7L2 at the hepatic silent GLUT4 gene. Axin2 is a wellknown target of WNT signalling and has been shown to be a direct target of TCF7L2 in human colon cancer cells [23]. We designed primers around TCF7L2 consensus sites approximately $2.5 \mathrm{~kb}$ upstream of the transcriptional start site (TSS) of Axin2 and used this region as our positive control (Fig. 3a, b). Using the enzymatic ChIP assay, we showed that TCF7L2 is highly enriched at this site (1\% input) (Fig. 3a), a finding that was later confirmed by the detection of a highly significant ChIP peak in an almost identical region (reads 601, FDR 1.5 $10^{-38}$ ) in our ChIP-Seq dataset (Fig. 3c).

Transcriptional targets of TCF $7 L 2$ in hepatocytes Illumina sequencing libraries were generated from input-control and TCF7L2-ChIP reactions and size-selected such that the final sequencing libraries were approximately 200 to $250 \mathrm{bp}$ in length (Fig. 3c) (GEO accession: GSE28782). We generated 40-base pair sequencing reads (input 29,226,566 reads; TCF7L2 ChIP 30,688,073 reads) and mapped them to the repeat-masked Rat Reference (RN4) Genome. Approximately $46 \%$ of the input and TCF7L2 reads aligned uniquely to the repeat-masked genome (ESM Table 2).

We detected a total of 2,119 ChIP peaks with CLC Genomics Workbench, using an FDR of $1 \%$. Peaks were subsequently divided into those located inside a reference gene and those within 5, 10, 50 and $500 \mathrm{~kb}$ of a TSS (ESM Table 3). Consistent with recent findings from $\beta$-catenin ChIP-Seq data in HCT116 colon cancer cells [24], there was a tendency for TCF7L2 to bind internally within gene boundaries. Overall, in our dataset, $36 \%$ (764 of 2119) of all binding events were inside a gene (Fig. 4a and ESM Table 4). There were 243 $(12 \%)$ independent binding events within $5 \mathrm{~kb}$ of a gene, 85 (4\%) within $10 \mathrm{~kb}, 286(14 \%)$ within $50 \mathrm{~kb}$ and $285(13 \%)$ within $500 \mathrm{~kb}$ (Fig. 4a and ESM Table 4). Cumulatively, a total of $65 \%(1,378$ of 2,119$)$ of all ChIP peaks in our dataset were within $50 \mathrm{~kb}$ of a gene (Fig. 4b and ESM Table 4). 

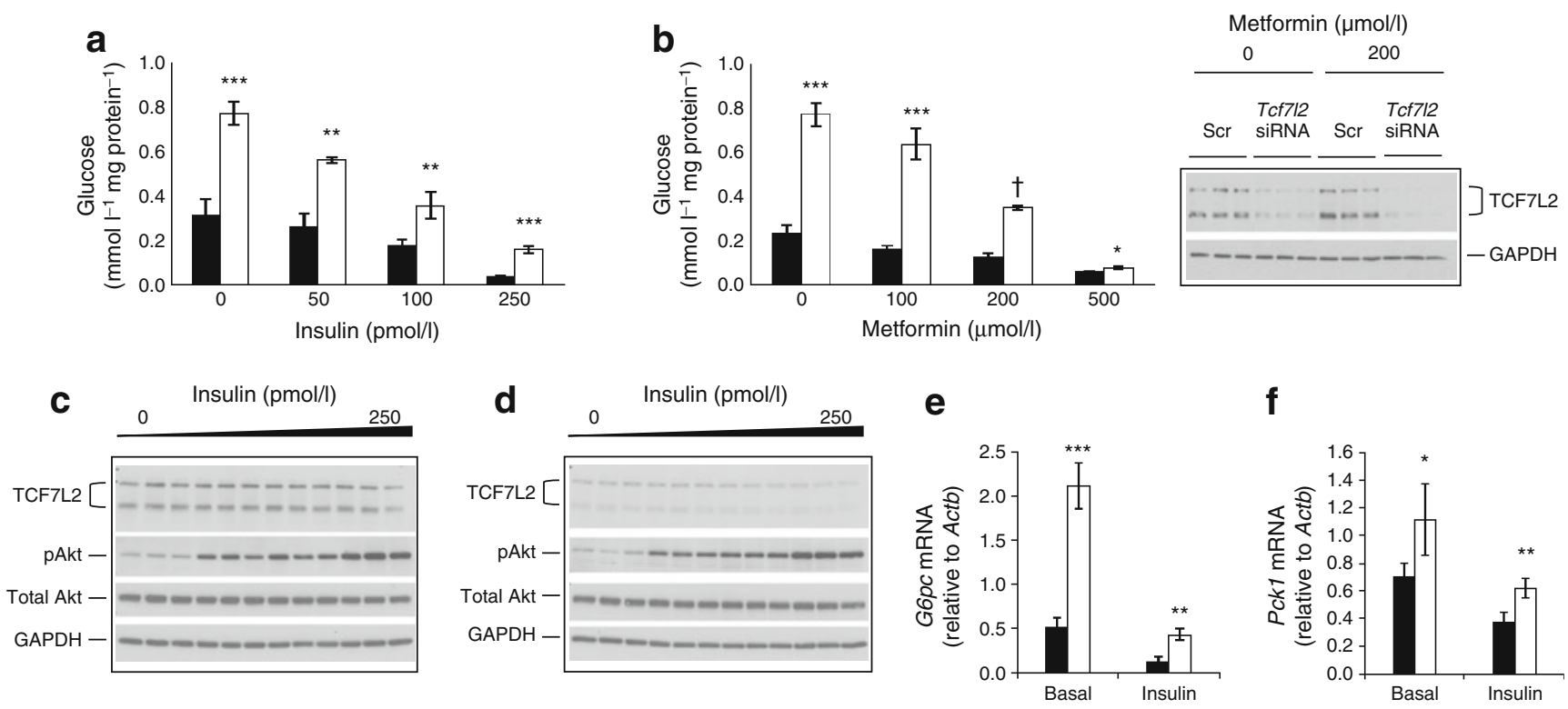

Fig. 2 Effect of TCF7L2 silencing on insulin- and metformin-mediated suppression of HGP in H4IIE cells. Insulin (a) and metformin (b) suppressed HGP in a dose-dependent manner, but TCF7L2 silencing did not affect the $\mathrm{IC}_{50}$ of insulin or metformin. However, absolute levels of HGP remained elevated owing to the significantly higher baseline HGP. Insulin sensitivity, as determined by pAKT levels, was found to be

Motif analysis of TCF7L2-enriched regions A search for the canonical (T/A-T/A-C-A-A-A-G) and the evolutionarily conserved (A-C/G-T/A-T-C-A-A-A-G) TCF7L2 motifs showed that $65 \%$ of all peaks $(1,388$ of 2,119$)$ had at least one copy of the canonical motif and $28 \%(589$ of 2,119$)$ had at least one copy of the long, evolutionarily conserved motif (ESM Table 5). To validate this search and to identify potentially unknown motifs in a subset of the TCF7L2 ChIP peaks, we performed a de novo motif search using MEMEChIP on a subset of our data. Searching for motifs between 6 and $15 \mathrm{bp}$ in length, we found a remarkable and highly consistent enrichment of the long, evolutionarily conserved TCF7L2 binding motif (Fig. 4c-f). Restricting the search to just 7 bp revealed only enrichment of the shorter canonical motif (not shown). We did not detect any other significant consensus sequences in the rat hepatocytes.

Functional analysis of TCF7L2 target genes Several known WNT target genes were clearly identified in our dataset, including Lef1, Axin2 and Ccnd1 (cyclin D1) (ESM Table 3). To gain an understanding of the overall function of the TCF7L2-bound genes in hepatocytes, we used Ingenuity Pathway Analysis (IPA, Redwood City, CA, USA). The input dataset consisted of 1,325 unique ChIP peaks (those within $50 \mathrm{~kb}$ only), of which 1,145 were mapped by the IPA software. We included all species for this analysis and stringent filters were set for all variables. Consistent with a recent report [25], the top two 'disease and disorder' categories from the IPA similar in scrambled (Scr) control cells (c) and TCF7L2-silenced cells (d). HGP remained elevated even at high concentrations of insulin, and this was associated with incomplete suppression of G6pc (e) and PckI (f) mRNA levels. Black bars, scrambled control; white bars, Tcf $7 l 2$ siRNA. Values are presented as mean $\pm \mathrm{SD} ;{ }^{*} p<0.05,{ }^{* *} p<0.01,{ }^{* * *} p<$ $0.001,{ }^{\dagger} p<0.0001$ vs control

analysis were 'genetic disorder' (644 genes) and 'metabolic disorder' (307 genes) (Table 1). In each of these categories, the diseases with the strongest representation were 'noninsulin dependent diabetes' (type 2 diabetes) (155 genes; $p=$ $\left.1.63 \times 10^{-10}\right)$ and 'diabetes mellitus' (245 genes; $p=7.4 \times$ $10^{-12}$ ), respectively (Table 1 and Table 2). Moreover, the top canonical signalling pathway revealed by our IPA analysis was 'type 2 diabetes signalling' (Table 3 ).

Because of the clear enrichment of our dataset with genes important for type 2 diabetes and metabolism, we inspected these genes to identify those that may be responsible for carbohydrate metabolism and/or HGP in hepatocytes. Of the 155 genes in the type 2 diabetes category, we identified several potentially important targets of TCF7L2 (Fig. 5). Of particular interest to the present study was the enrichment seen at Pckl, Pdk4 and Fbpl. Multiple peaks were observed close to the $P c k 1$ gene $\left(-11 \mathrm{~kb} \mathrm{3}\right.$ ' from TSS) and one in the $5^{\prime}$ promoter region $(-1,125 \mathrm{bp}$ from TSS). A significant ChIP peak was also located in intron 3 of $F b p l$, which is located $15 \mathrm{~kb}$ from the next nearest gene, which is an additional fructose 1,6bisphosphatase locus, Fbp2. Both the Pck1 and Fbpl ChIP peaks included at least one copy ( $F b p l$ two copies) of the consensus TCF7L2 binding motif within the flanking regions.

We also observed enrichment at several other genes critical for insulin signalling and hepatic glucose metabolism. These included Irs1 and Irs2, Akt2, Adipor1, Prkcz, 
a

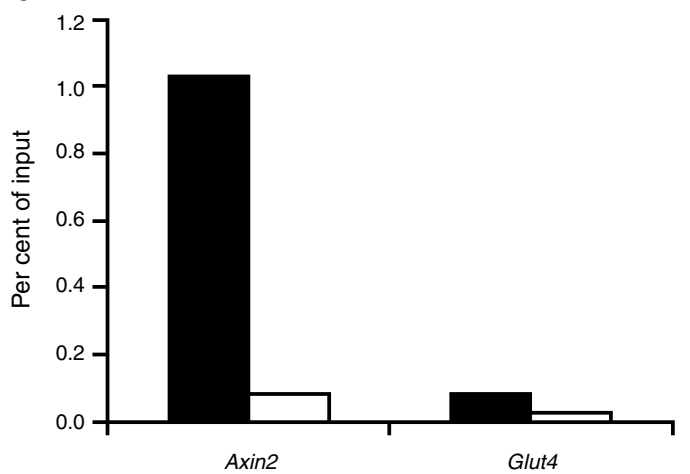

b

$98,300,000$
C

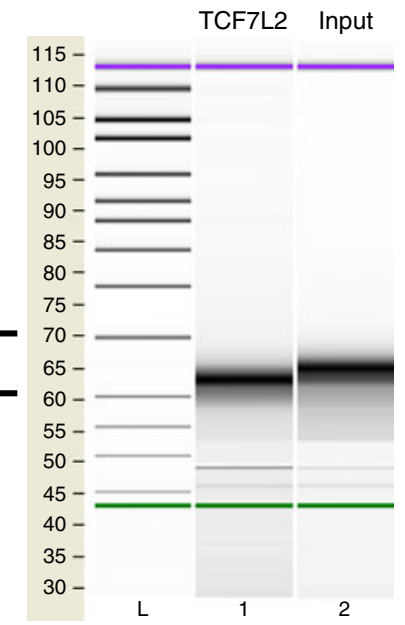

$98,310,000$

$98,320,000$

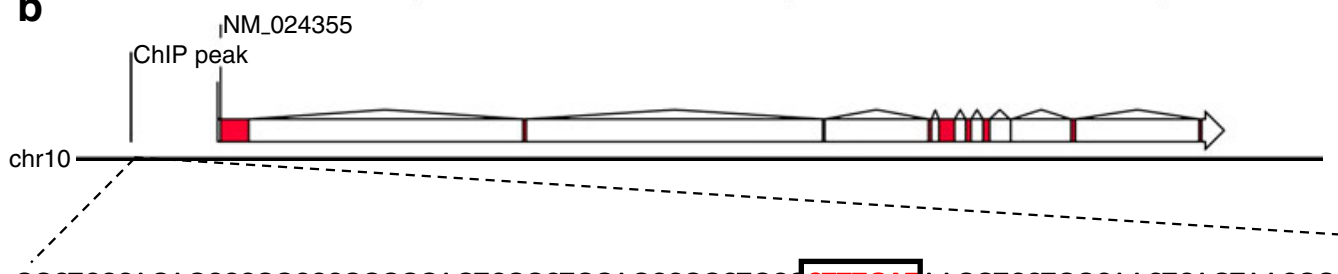

GGCTCCCAGAGCCCGGCCCGGGGGAGTCGGCTGGAGCCGGCTGC CTTTGATAAGGTCCTGGCAACTCAGTAACGGCCCGAGAACCGGGAAATAAAAA TAAGCCGCCGATCGCGATGGATTTCGGGGCCACCCGGAGGCCGAGGCGTCCGCCTCCCCAAAGGAGAGCTCTGCTGTAAAAGGTGGGGTCGGAGGCTT AGTCCCGCCGCAGTCGCTTCCAAGAAACCTCGGTGCGGCCGGGGGCGGTTGGCANNNNNNNNNNNNNNNNNNNNNNNNNNNNNNNNNNAGCAG GCCCGGGTAGTAGAGGGGTGCGGATTGCACAGCCCCCAAAAGGGGCGCTGGAGCGCGCTGGGCTGGCCCGGGTGCGGCTCGCATACCTCCCTTCCAGG ACCGTGCGCTCGGCTCGCG CTTTGAAGTGCACAGTTAAATCCACAGCGCAGTTTTTGTTGGAAGCGCCCGGAGGACATTTGGTGCGCCTAGTGACTGC TGGAAACTGGACCTGGGTTGAAANNNNNNNNNNNNNNNNNNNNNNNNNNNNNNNNNNNNNNNNNAGGCCCACTCGGATCTTTTGCATAAAAGG GGCAGCGGGGCGCACGATCTCCCCCCCTCCCTCCCCAGCGCAGAGCTCGCTCTCGCCTCGGGATCGTTGTTTTCCGCGCACCCCCTCCTCCGCCTCTGCGA GACTCCTCATGTTCCCCANNNNNNNNNNNNNNNNNNNNNNNNNNN

Fig. 3 ChIP of TCF7L2 in H4IIE cells and ChIP-Seq library preparation. a The WNT target gene Axin2 was used as a positive control region for the initial ChIP assay and was significantly bound by TCF7L2 in the 5' promoter region. Glut4, also known as Slc2a4, is hepatic-silent and was used as a negative control region in the ChIP assay. Black bars, TCF7L2 ChIP; white bars, IgG ChIP. b A figure from our CLC Genomics Workbench analysis shows a ChIP-Seq peak corresponding to the same site used as the positive control in the ChIP

Gys2 and Cptla. Of these, we found that all except Cpt1a contain at least one copy of the canonical TCF7L2 motif and that Akt 2 contains a canonical and an evolutionarily conserved TCF7L2 recognition site. We confirmed a number of these peaks independently using ChIP assays followed by quantitative RT-PCR (Fig. 5).

\section{Discussion}

In the current study, we have demonstrated that silencing of TCF7L2, alone and selectively, is sufficient to markedly increase HGP from gluconeogenic precursors (lactate/ pyruvate) in hepatocytes in vitro. Using a wholegenome ChIP-Seq approach, we have also shown here assay; it validates the strong binding of the Axin2 gene by TCF7L2 and confirms the specificity of our antibody in the ChIP-Seq experiments. The ChIP-Seq peak and flanking region are shown and the ChIP assay positive control PCR product is highlighted (bold and underlined). Red text in boxes indicates putative TCF7L2 binding sites. c Final Illumina ChIP-Seq libraries were approximately 200 to $250 \mathrm{bp}$ in length, as shown by the Bioanalyzer gel image (Agilent Technologies, Santa Clara, CA, USA)

for the first time that TCF7L2 binds to a number of genes important for glucose metabolism and insulin signalling. Together, these data suggest that TCF7L2 plays an important role in the regulation of hepatic glucose metabolism.

Polymorphisms within TCF7L2 have consistently been associated with an elevated risk of type 2 diabetes [2]. Numerous studies have suggested a strong link between TCF7L2 and pancreatic beta cell function in vivo and in vitro $[5,7,26]$. However, there is also evidence to suggest that variants in TCF7L2 may augment basal HGP and impair the ability of insulin to inhibit hepatic gluconeogenesis. Pilgaard and colleagues [8] showed that basal HGP was elevated and failed to be suppressed normally during a hyperinsulinaemic-euglycaemic clamp in T allele carriers, a 
Fig. 4 a TCF7L2 preferentially binds close to target genes and $36 \%$ of all ChIP-Seq peaks were within gene boundaries (including introns and exons). b Cumulatively, $65 \%$ of all TCF7L2 binding events were within $50 \mathrm{~kb}$ of the TSS of annotated genes. De novo TCF7L2 motif analysis of all ChIP-Seq peaks in chromosome 1 (total peaks 191, sites found 77, $E$ value $\left.5.3 \times 10^{-65}\right)(\mathbf{c})$, chromosome 5 (total peaks 91 , sites found $53, E$ value $1.0 \times 10^{-55}$ ) (d), chromosome 10 (total peaks 100 , sites found 57 , $E$ value $6.9 \times 10^{-50}$ ) (e) and chromosome 15 (total peaks 42 , sites found $37, E$ value $6.9 \times$ $10^{-50}$ ) (f) was performed using MEME-ChIP. This analysis demonstrated the significant enrichment of our ChIP-Seq dataset with peaks containing the conserved TCF7L2 binding motifs
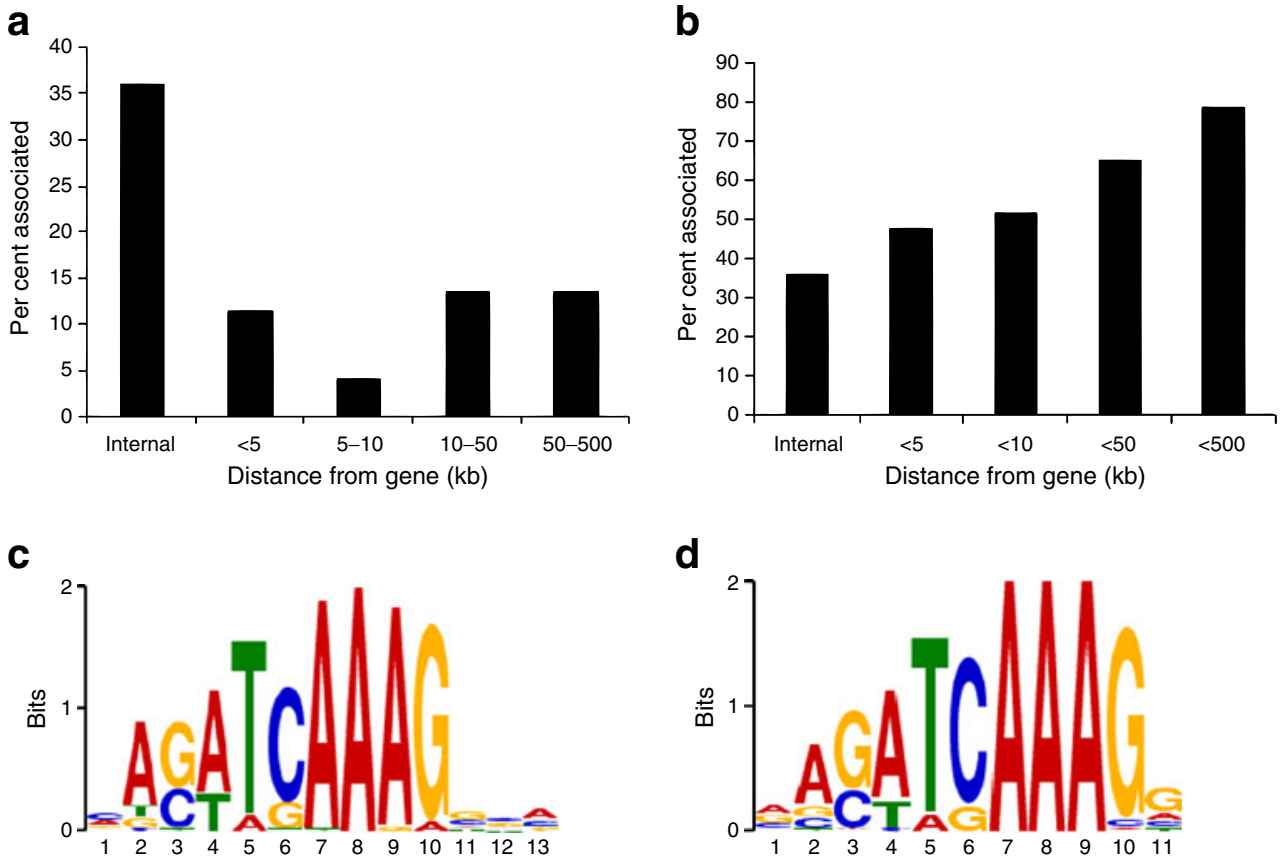

d

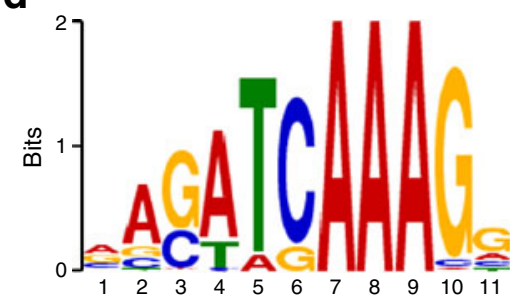

e

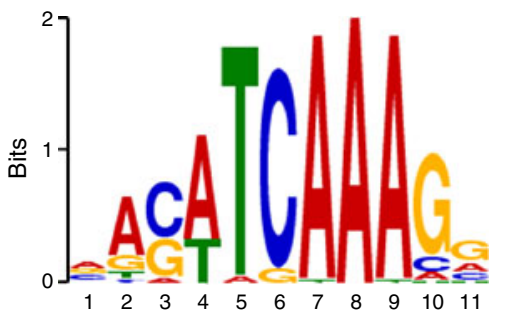

f

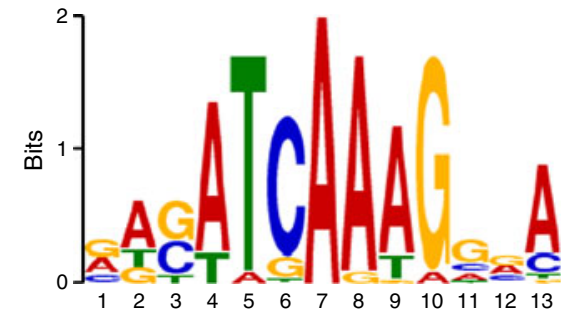

finding confirmed in larger studies [7]. Elevated hepatic insulin resistance in $\mathrm{T}$ allele carriers has also been documented in a Danish twin cohort [6] and in patients with nonalcoholic fatty liver disease [27]. Our findings support a role for TCF7L2 in regulating hepatic gluconeogenesis and suggest that this regulation may occur at the transcriptional level. However, the ability of insulin to inhibit HGP from gluconeogenesis (i.e. $\mathrm{IC}_{50}$ ) was preserved when TCF7L2 was silenced. Because of the marked increase in basal HGP, the absolute rate of HGP for any given insulin concentration remained elevated in hepatocytes treated with $T c f F 7 l 2$ siRNA compared with those treated with scrambled siRNA.

Although we used an in vitro model, our results, if extrapolated to an in vivo system, suggest that the $\mathrm{T}$ allele at rs7903146 may play a role in fasting as well as postprandial hyperglycaemia, which would be consistent with results from studies employing the euglycaemic clamp technique [8]. The precise functional impact of the T allele in vitro or in vivo is uncertain, but it is suspected to alter the expression and/or splicing of TCF7L2. Some studies have shown reduced expression of TCF7L2 in diabetic islets [28], whereas others have noted a marked increase in TCF7L2 mRNA in T allele carriers [7], or no change at all [29]. The current study did not examine the relationship between rs7903146 and HGP, but our results suggest that TCF7L2 suppresses the expression of genes involved in gluconeogenesis and that reduced TCF7L2 protein levels lead to enhanced HGP. It is not known whether rs7903146
Table 1 IPA of the ChIP-Seq data, showing the top five functional annotations for the metabolic disease category

\begin{tabular}{lllr}
\hline & Functional annotation & $p$ value & Molecules $(n)$ \\
\hline Metabolic disease & Diabetes mellitus & $7.44 \times 10^{-12}$ & 245 \\
Metabolic disease & Non-insulin-dependent diabetes mellitus & $1.63 \times 10^{-10}$ & 155 \\
Metabolic disease & Dyslipidaemia & $3.15 \times 10^{-5}$ & 12 \\
Metabolic disease & Insulin-dependent diabetes mellitus & $4.40 \times 10^{-5}$ & 108 \\
Metabolic disease & Insulin resistance of mice & $1.99 \times 10^{-4}$ & 15 \\
\hline
\end{tabular}


Table 2 IPA of the ChIP-Seq data, showing the top five functional annotations for the genetic disorder category

\begin{tabular}{lllc}
\hline & Functional annotation & $p$ value & Molecules $(n)$ \\
\hline Genetic disorder & Non-insulin-dependent diabetes mellitus & $1.63 \times 10^{-10}$ & 155 \\
Genetic disorder & Bipolar disorder & $3.25 \times 10^{-10}$ & 128 \\
Genetic disorder & Coronary artery disease & $2.16 \times 10^{-9}$ & 136 \\
Genetic disorder & Inflammatory bowel disease & $1.59 \times 10^{-7}$ & 130 \\
Genetic disorder & Crohn's disease & $2.45 \times 10^{-7}$ & 122 \\
\hline
\end{tabular}

affects the expression of TCF7L2 in the liver in vivo, but our results may support the hypothesis that the $\mathrm{T}$ allele reduces abundance of the protein. In humans, the $\mathrm{T}$ allele is associated with elevated HGP [8]. Further studies examining the impact of the $\mathrm{T}$ allele on TCF7L2 expression and function in the liver are needed.

Glucose production from hepatocytes is controlled by the expression and/or activity of several rate-limiting enzymes [30]. The genes encoding three key gluconeogenic enzymes, Pckl, Fbp1 and G6pc, were all significantly upregulated by TCF7L2 silencing in the present study. We believe that the increased expression of these three genes played a major role in the increase of HGP observed by us. Moreover, it is possible that TCF7L2 directly regulates the expression of Pckl and Fbpl, as we detected significant binding events at both of these genes in our ChIP-Seq dataset. Using IPA analysis, we also showed that TCF7L2 binds to genes known to be important for hepatic glucose metabolism. These genes included Irs1, Adiporl, Gys2, Cpt1a and Pdk4. Several of these genes may have a direct role in hepatic glucose metabolism in vivo. Glycogen synthase-2, which is produced exclusively in the liver, catalyses the rate-limiting step in the synthesis of glycogen, and defects in this protein lead to glycogen storage disease (type 0), a condition associated with elevated postprandial hyperglycaemia and fasting hypoglycaemia [31]. Pyruvate dehydrogenase kinase 4 (PDK4) is an inhibitor of the pyruvate dehydrogenase complex (PDC), which catalyses the oxidative decarboxylation of pyruvate to acetyl-CoA [32]. PDK4 enhances hepatic gluconeogenesis through inhibition of the PDC complex and thus preservation of pyruvate for gluconeogenesis, and is upregulated during starvation and in insulin-resistant states [32]. CPT1A, again expressed exclusively in the liver, plays a key role in $\beta$ -

Table 3 Genes represented by the type 2 diabetes signalling category following IPA analysis

\begin{tabular}{|c|c|c|c|}
\hline Symbol & Entrez Gene name & Location & Type \\
\hline Acsl5 & Acyl-CoA synthetase long-chain family member 5 & Cytoplasm & Enzyme \\
\hline Adipoq & Adiponectin, C1Q and collagen domain containing & Extracellular & Other \\
\hline Adipor1 & Adiponectin receptor 1 & Membrane & Receptor \\
\hline Akt2 & v-akt murine thymoma viral oncogene homolog 2 & Cytoplasm & Kinase \\
\hline Atm & Ataxia telangiectasia mutated & Nucleus & Kinase \\
\hline$I k b k b$ & Inhibitor of kappa light polypeptide gene enhancer in B cells, kinase beta & Cytoplasm & Kinase \\
\hline Irs 1 & Insulin receptor substrate 1 & Cytoplasm & Other \\
\hline $\operatorname{Irs} 2$ & Insulin receptor substrate 2 & Cytoplasm & Other \\
\hline Nfkbia & Nuclear factor of kappa light polypeptide gene enhancer in B cells inhibitor, alpha & Cytoplasm & Other \\
\hline$P i k 3 c 2 b$ & Phosphoinositide-3-kinase, class 2 , beta polypeptide & Cytoplasm & Kinase \\
\hline Pik3r4 & Phosphoinositide-3-kinase, regulatory subunit 4 & Cytoplasm & Other \\
\hline Pkm2 & Pyruvate kinase, muscle & Cytoplasm & Kinase \\
\hline Prkag2 & Protein kinase, AMP-activated, gamma 2 non-catalytic subunit & Cytoplasm & Kinase \\
\hline Prkca & Protein kinase $\mathrm{C}$, alpha & Cytoplasm & Kinase \\
\hline Prkcd & Protein kinase $\mathrm{C}$, delta & Cytoplasm & Kinase \\
\hline Prkcz & Protein kinase $\mathrm{C}$, zeta & Cytoplasm & Kinase \\
\hline Prkd3 & Protein kinase D3 & Unknown & Kinase \\
\hline$S l c 27 a 3$ & Solute carrier family 27 (fatty acid transporter), member 3 & Cytoplasm & Transporter \\
\hline Slc27a5 & Solute carrier family 27 (fatty acid transporter), member 5 & Cytoplasm & Transporter \\
\hline Smpd3 & Sphingomyelin phosphodiesterase 3, neutral membrane (neutral sphingomyelinase II) & Cytoplasm & Enzyme \\
\hline Socs 1 & Suppressor of cytokine signalling 1 & Cytoplasm & Other \\
\hline Socs3 & Suppressor of cytokine signalling 3 & Cytoplasm & Other \\
\hline
\end{tabular}




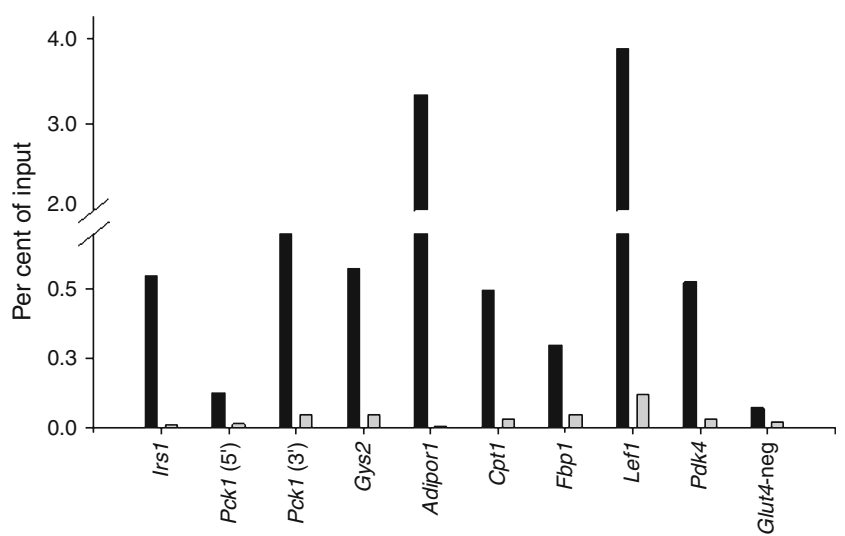

Fig. 5 Independent ChIP assays were performed to confirm the binding of TCF7L2 to several genes important for carbohydrate metabolism and insulin signalling in hepatocytes. An independent preparation of chromatin was used for this experiment and SYBR Green primers were designed according to the location of the ChIP-Seq peaks detected in our CLC Genomics Workbench analysis. Black bars, TCF7L2 ChIP; grey bars, Myc-tag ChIP. Glut4, also known as Slc2a4 was used as a negative control region

oxidation of fatty acids in the mitochondria; its selective inhibition reduces gluconeogenesis and improves glucose homeostasis in vivo [33]. Adiponectin suppresses HGP independently of insulin in primary hepatocytes [34,35] and adiponectin receptors have been shown to be reduced in mouse models of diabetes [36]. Finally, signals from IRS1 (and IRS2) are critical for the maintenance and control of HGP; silencing of both together leads to severe and persistent hyperglycaemia [37]. Clearly, the transcriptional activity of TCF7L2 could have significant implications for the regulation of HGP, insulin resistance and type 2 diabetes.

We cannot exclude the possibility that the robust changes in expression of genes involved in gluconeogenesis were part of a more global phenotypic shift in the hepatocytes, consistent with a role for TCF7L2 in metabolic zonation. The WNT/ $\beta$-catenin pathway is a major determinant of the metabolic fate of hepatocytes [9, 38-40]. In this regard, several other pathways, including those responsible for amino acid, ammonia and drug metabolism, might also have been altered in our experimental model. Notably, we did observe significant ChIP-Seq peaks at several genes involved in the regulation of these pathways, including Oat (glutamine synthesis), Slcla2 (glutamine synthesis), Cps1 (urea formation) and $R h b g$ (ammonia transport). Interestingly, these genes, in addition to Pckl, Fbpl and G6pc, have also been identified in $\beta$-catenin-activated hepatocytes $[13,14,41]$. The effect of TCF7L2 silencing on Hnf4a expression may indeed suggest that TCF7L2 silencing induced a phenotypic switch in the hepatocytes. Hepatocyte nuclear factor $4 \alpha(\mathrm{HNF} 4 \alpha)$ is an important determinant of the periportal (gluconeogenic) transcriptional program.
Hepatic $\mathrm{HNF} 4 \alpha$ deficiency induces periportal production of glutamine synthetase and several other pericentral enzymes [42], acting through cross-talk with the WNT pathway [43]. The mechanisms underlying the interaction between WNT and HNF $4 \alpha$ are poorly understood, but may converge on the WNT transcription factor lymphoid enhancer binding factor 1 (LEF1) [43].

Over $60 \%$ of the ChIP-Seq peaks detected in our analysis had at least one copy of the short consensus sequence, either inside the peak or within proximal $5^{\prime}$ or $3^{\prime}$ flanking regions. Almost one third of the peaks had the long, evolutionarily conserved motif within the flanking regions. These data are consistent with several studies documenting chromatin occupancy of TCF7L2 in colon cancer cells [23-25]. An unbiased de novo search for TCF7L2 binding motifs did not reveal any novel TCF7L2 recognition sequences. It is possible that motifs for other transcription factors (nuclear factor-1, HNF4, peroxisome proliferator-activated receptor $\gamma$ ) were located within or surrounding our ChIP-Seq peaks, as has been shown by others [23], but we did not specifically test this hypothesis in the current study. Peaks not containing a recognisable motif may represent protein $\times$ protein interactions between TCF7L2 and other factors directly recruited to these areas.

We have shown that TCF7L2 is preferentially located inside gene boundaries (intragenic) and, indeed, $65 \%$ of all peaks were within $50 \mathrm{~kb}$ of the TSS of a gene or within the gene enhancer region, findings that again are consistent with previously published data [24]. Hatzis et al. [23] commented that TCF7L2 had a tendency to cluster $\left(5^{\prime}, 3^{\prime}\right.$, internal) around target genes. While we did not formally search for this type of binding pattern, several TCF7L2 target genes (e.g. Lef1 and Pck1) had multiple ChIP peaks across the gene and flanking regions. These data are consistent with the concept, suggested by others [23], that this binding pattern supports an open chromatin domain or provides a more accurate $\beta$-catenin sensor mechanism.

In summary, we have provided the first evidence for the role of TCF7L2 in the regulation of HGP via the gluconeogenesis pathway in vitro. Although more work is needed to define the precise role of this protein in hepatic glucose metabolism in vivo, our data provide a possible link between genetic association studies of TCF7L2 with type 2 diabetes and a physiological function that is highly relevant and disturbed in this common metabolic disease that affects $8 \%$ of the US population [44].

Acknowledgements This work was supported by a San Antonio Area Foundation research grant to L. Norton. We would like to thank W. Wakeland, C. Arana and C. Liang at the Department of Immunology, UT Southwestern Medical Center (Dallas, TX, USA) for Illumina sequencing services. 
Contribution statement LN conceived and designed the experiments, researched data, wrote the manuscript, contributed to discussion, and reviewed and edited the manuscript. MF, FFM and DW were involved in the analysis and interpretation of the data and in critically revising the article. MAG, CPJ and RAD conceived and designed the experiments, contributed to discussion, and critically reviewed and edited the manuscript. All authors approved the final version of this manuscript.

Duality of interest The authors declare that there is no duality of interest associated with this manuscript.

\section{References}

1. Duggirala R, Blangero J, Almasy L et al (1999) Linkage of type 2 diabetes mellitus and of age at onset to a genetic location on chromosome $10 \mathrm{q}$ in Mexican Americans. Am J Hum Genet 64:1127-1140

2. Grant SF, Thorleifsson G, Reynisdottir I et al (2006) Variant of transcription factor 7-like 2 (TCF7L2) gene confers risk of type 2 diabetes. Nat Genet 38:320-323

3. Cauchi S, El Achhab Y, Choquet $\mathrm{H}$ et al (2007) TCF7L2 is reproducibly associated with type 2 diabetes in various ethnic groups: a global meta-analysis. J Mol Med 85:777-782

4. Clevers H (2006) Wnt/beta-catenin signaling in development and disease. Cell 127:469-480

5. Shu L, Sauter NS, Schulthess FT, Matveyenko AV, Oberholzer J, Maedler K (2008) Transcription factor 7-like 2 regulates beta-cell survival and function in human pancreatic islets. Diabetes 57:645-653

6. Wegner L, Hussain MS, Pilgaard K et al (2008) Impact of TCF7L2 rs7903146 on insulin secretion and action in young and elderly Danish twins. J Clin Endocrinol Metab 93:4013-4019

7. Lyssenko V, Lupi R, Marchetti P et al (2007) Mechanisms by which common variants in the TCF7L2 gene increase risk of type 2 diabetes. J Clin Invest 117:2155-2163

8. Pilgaard K, Jensen CB, Schou JH et al (2009) The T allele of rs7903146 TCF7L2 is associated with impaired insulinotropic action of incretin hormones, reduced $24 \mathrm{~h}$ profiles of plasma insulin and glucagon, and increased hepatic glucose production in young healthy men. Diabetologia 52:1298-1307

9. Gebhardt R, Hovhannisyan A (2010) Organ patterning in the adult stage: the role of Wnt/beta-catenin signaling in liver zonation and beyond. Dev Dyn 239:45-55

10. Jungermann K (1987) Metabolic zonation of liver parenchyma: significance for the regulation of glycogen metabolism, gluconeogenesis, and glycolysis. Diabetes Metab Rev 3:269-293

11. Katz N, Teutsch HF, Jungermann K, Sasse D (1977) Heterogeneous reciprocal localization of fructose-1,6-bisphosphatase and of glucokinase in microdissected periportal and perivenous rat liver tissue. FEBS Lett 83:272-276

12. Torre C, Perret C, Colnot S (2011) Transcription dynamics in a physiological process: beta-catenin signaling directs liver metabolic zonation. Int J Biochem Cell Biol 43:271-278

13. Benhamouche S, Decaens T, Godard C et al (2006) Apc tumor suppressor gene is the "zonation-keeper" of mouse liver. Dev Cell 10:759-770

14. Braeuning A, Ittrich C, Kohle C et al (2006) Differential gene expression in periportal and perivenous mouse hepatocytes. FEBS J 273:5051-5061

15. de Raemy-Schenk AM, Trouble S, Gaillard P et al (2006) A cellular assay for measuring the modulation of glucose production in H4IIE cells. Assay Drug Dev Technol 4:525-533

16. Duong DT, Waltner-Law ME, Sears R, Sealy L, Granner DK (2002) Insulin inhibits hepatocellular glucose production by utilizing liver-enriched transcriptional inhibitory protein to disrupt the association of CREB-binding protein and RNA polymerase II with the phosphoenolpyruvate carboxykinase gene promoter. J Biol Chem 277:32234-32242

17. Gao Y, Walder K, Sunderland T et al (2003) Elevation in Tanis expression alters glucose metabolism and insulin sensitivity in H4IIE cells. Diabetes 52:929-934

18. Korinek V, Barker N, Morin PJ et al (1997) Constitutive transcriptional activation by a $\beta$-catenin-Tcf complex in $\mathrm{APC}^{-/}$ colon carcinoma. Science 275:1784-1787

19. Norton L, Parr T, Chokkalingam K et al (2008) Calpain-10 gene and protein expression in human skeletal muscle: effect of acute lipid-induced insulin resistance and type 2 diabetes. J Clin Endocrinol Metab 93:992-998

20. Tsedensodnom O, Koga H, Rosenberg SA et al (2011) Identification of $\mathrm{T}$ cell factor- 4 isoforms that contribute to the malignant phenotype of hepatocellular carcinoma cells. Exp Cell Res 317:920 931

21. Osmark P, Hansson O, Jonsson A, Ronn T, Groop L, Renstrom E (2009) Unique splicing pattern of the TCF7L2 gene in human pancreatic islets. Diabetologia 52:850-854

22. Bailey TL, Elkan C (1994) Fitting a mixture model by expectation maximization to discover motifs in biopolymers. Proc Int Conf Intell Syst Mol Biol 2:28-36

23. Hatzis P, van der Flier LG, van Driel MA et al (2008) Genome-wide pattern of TCF7L2/TCF4 chromatin occupancy in colorectal cancer cells. Mol Cell Biol 28:2732-2744

24. Bottomly D, Kyler SL, McWeeney SK, Yochum GS (2010) Identification of $\beta$-catenin binding regions in colon cancer cells using ChIP-Seq. Nucleic Acids Res 38:5735-5745

25. Zhao J, Schug J, Li M, Kaestner KH, Grant SF (2010) Diseaseassociated loci are significantly over-represented among genes bound by transcription factor 7-like 2 (TCF7L2) in vivo. Diabetologia 53:2340-2346

26. Liu Z, Habener JF (2008) Glucagon-like peptide-1 activation of TCF7L2-dependent Wnt signaling enhances pancreatic beta cell proliferation. J Biol Chem 283:8723-8735

27. Musso G, Gambino R, Pacini G, Pagano G, Durazzo M, Cassader M (2009) Transcription factor 7-like 2 polymorphism modulates glucose and lipid homeostasis, adipokine profile, and hepatocyte apoptosis in NASH. Hepatology 49:426435

28. Shu L, Matveyenko AV, Kerr-Conte J, Cho JH, McIntosh CH, Maedler K (2009) Decreased TCF7L2 protein levels in type 2 diabetes mellitus correlate with downregulation of GIP- and GLP-1 receptors and impaired beta-cell function. Hum Mol Genet 18:23882399

29. Elbein SC, Chu WS, Das SK et al (2007) Transcription factor 7 like 2 polymorphisms and type 2 diabetes, glucose homeostasis traits and gene expression in US participants of European and African descent. Diabetologia 50:1621-1630

30. Nordlie RC, Foster JD, Lange AJ (1999) Regulation of glucose production by the liver. Annu Rev Nutr 19:379-406

31. Orho M, Bosshard NU, Buist NR et al (1998) Mutations in the liver glycogen synthase gene in children with hypoglycemia due to glycogen storage disease type 0. J Clin Invest 102:507515

32. Sugden MC (2003) PDK4: a factor in fatness? Obes Res 11:167169

33. Conti R, Mannucci E, Pessotto P et al (2011) Selective reversible inhibition of liver carnitine palmitoyl-transferase 1 by teglicar reduces gluconeogenesis and improves glucose homeostasis. Diabetes 60:644-651

34. Zhou H, Song X, Briggs M et al (2005) Adiponectin represses gluconeogenesis independent of insulin in hepatocytes. Biochem Biophys Res Commun 338:793-799 
35. Yoon YS, Ryu D, Lee MW, Hong S, Koo SH (2009) Adiponectin and thiazolidinedione targets CRTC2 to regulate hepatic gluconeogenesis. Exp Mol Med 41:577-583

36. Inukai K, Nakashima Y, Watanabe $M$ et al (2005) Regulation of adiponectin receptor gene expression in diabetic mice. Am J Physiol Endocrinol Metab 288:E876-E882

37. Dong X, Park S, Lin X, Copps K, Yi X, White MF (2006) Irs1 and Irs2 signaling is essential for hepatic glucose homeostasis and systemic growth. J Clin Invest 116:101-114

38. Cadoret A, Ovejero C, Terris B et al (2002) New targets of beta-catenin signaling in the liver are involved in the glutamine metabolism. Oncogene 21:8293-8301

39. Loeppen S, Schneider D, Gaunitz F et al (2002) Overexpression of glutamine synthetase is associated with beta-catenin-mutations in mouse liver tumors during promotion of hepatocarcinogenesis by phenobarbital. Cancer Res 62:5685-5688

40. Nicholes K, Guillet S, Tomlinson E et al (2002) A mouse model of hepatocellular carcinoma: ectopic expression of fibroblast growth factor 19 in skeletal muscle of transgenic mice. Am J Pathol 160:2295-2307

41. Chafey P, Finzi L, Boisgard R et al (2009) Proteomic analysis of beta-catenin activation in mouse liver by DIGE analysis identifies glucose metabolism as a new target of the Wnt pathway. Proteomics 9:3889-3900

42. Stanulovic VS, Kyrmizi I, Kruithof-de Julio M et al (2007) Hepatic HNF4alpha deficiency induces periportal expression of glutamine synthetase and other pericentral enzymes. Hepatology 45:433-444

43. Colletti M, Cicchini C, Conigliaro A et al (2009) Convergence of Wnt signaling on the HNF4alpha-driven transcription in controlling liver zonation. Gastroenterology 137:660672

44. Cowie CC, Rust KF, Byrd-Holt DD et al (2006) Prevalence of diabetes and impaired fasting glucose in adults in the U.S. population: National Health And Nutrition Examination Survey 1999-2002. Diabetes Care 29:1263-1268 